\title{
Front Matter: Volume 10708
}

, "Front Matter: Volume 10708," Proc. SPIE 10708, Millimeter, Submillimeter, and Far-Infrared Detectors and Instrumentation for Astronomy IX, 1070801 (1 August 2018); doi: 10.1117/12.2506058

SDIE Event: SPIE Astronomical Telescopes + Instrumentation, 2018, Austin, Texas, SPIE. United States 


\title{
PROCEEDINGS OF SPIE
}

\section{Millimeter, Submillimeter, and Far-Infrared Detectors and Instrumentation for Astronomy IX}

\author{
Jonas Zmuidzinas \\ Jian-Rong Gao \\ Editors
}

\section{2-15 June 2018 \\ Austin, Texas, United States}

Sponsored by

SPIE

Cosponsored by

4D Technology (United States) • Andor Technology, Ltd. (United Kingdom) • Astronomical Consultants \& Equipment, Inc. (United States) • Giant Magellan Telescope (Chile) • GPixel, Inc. (China) • Harris Corporation (United States) • Materion Corporation (United States) • Optimax Systems, Inc. (United States) • Princeton Infrared Technologies (United States) • Symétrie (France) • Teledyne Technologies, Inc. (United States) • Thirty Meter Telescope (United States)

\section{Cooperating Organizations}

European Space Organisation • National Radio Astronomy Observatory (United States) • Science \& Technology Facilities Council (United Kingdom) • Canadian Astronomical Society (Canada) • Canadian Space Association ASC (Canada) • Royal Astronomical Society (United Kingdom) • Association of Universities for Research in Astronomy (United States) • American Astronomical Society (United States) • Australian Astronomical Observatory (Australia) • European Astronomical Society (Switzerland)

Published by

SPIE

\section{Volume 10708}

Part One of Two Parts 
The papers in this volume were part of the technical conference cited on the cover and title page. Papers were selected and subject to review by the editors and conference program committee. Some conference presentations may not be available for publication. Additional papers and presentation recordings may be available online in the SPIE Digital Library at SPIEDigitallibrary.org.

The papers reflect the work and thoughts of the authors and are published herein as submitted. The publisher is not responsible for the validity of the information or for any outcomes resulting from reliance thereon.

Please use the following format to cite material from these proceedings:

Author(s), "Title of Paper," in Millimeter, Submillimeter, and Far-Infrared Detectors and Instrumentation for Astronomy IX, edited by Jonas Zmuidzinas, Jian-Rong Gao, Proceedings of SPIE Vol. 10708 (SPIE, Bellingham, WA, 2018) Seven-digit Article CID Number.

ISSN: 0277-786X

ISSN: 1996-756X (electronic)

ISBN: 9781510619692

ISBN: 9781510619708 (electronic)

Published by

SPIE

P.O. Box 10, Bellingham, Washington 98227-0010 USA

Telephone +1 3606763290 (Pacific Time) · Fax +1 3606471445

SPIE.org

Copyright (C) 2018, Society of Photo-Optical Instrumentation Engineers.

Copying of material in this book for internal or personal use, or for the internal or personal use of specific clients, beyond the fair use provisions granted by the U.S. Copyright Law is authorized by SPIE subject to payment of copying fees. The Transactional Reporting Service base fee for this volume is $\$ 18.00$ per article (or portion thereof), which should be paid directly to the Copyright Clearance Center (CCC), 222 Rosewood Drive, Danvers, MA 01923. Payment may also be made electronically through CCC Online at copyright.com. Other copying for republication, resale, advertising or promotion, or any form of systematic or multiple reproduction of any material in this book is prohibited except with permission in writing from the publisher. The CCC fee code is 0277 $786 \mathrm{X} / 18 / \$ 18.00$.

Printed in the United States of America.

Publication of record for individual papers is online in the SPIE Digital Library.

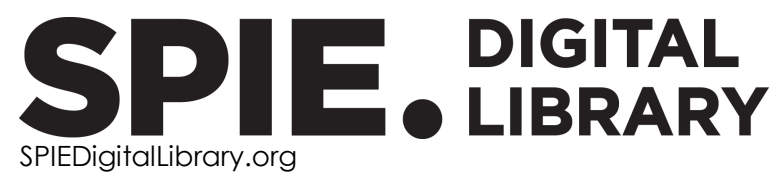

Paper Numbering: Proceedings of SPIE follow an e-First publication model. A unique citation identifier (CID) number is assigned to each article at the time of publication. Utilization of CIDs allows articles to be fully citable as soon as they are published online, and connects the same identifier to all online and print versions of the publication. SPIE uses a seven-digit CID article numbering system structured as follows:

- The first five digits correspond to the SPIE volume number.

- The last two digits indicate publication order within the volume using a Base 36 numbering system employing both numerals and letters. These two-number sets start with $00,01,02,03,04$, 05, 06, 07, 08, 09, 0A, OB ... 0Z, followed by 10-1Z, 20-2Z, etc. The CID Number appears on each page of the manuscript. 


\title{
Contents
}

\author{
ix Authors \\ xvii Conference Committee
}

\section{Part One}

\section{CMB INSTRUMENTS I}

1070805 BFORE: a CMB balloon payload to measure reionization, neutrino mass, and cosmic inflation [10708-4]

1070806 The primordial inflation polarization explorer (PIPER): current status and performance of the first flight [10708-5]

1070807 BICEP Array: a multi-frequency degree-scale CMB polarimeter [10708-49]

\section{OPTICS I}

10708 OD Development of large-diameter flat mesh-lenses for millimetre wave instrumentation [10708-11]

10708 OE Design and development of a polarization modulator unit based on a continuous rotating halfwave plate for LiteBIRD [10708-12]

10708 OF Aerogel scattering filters for cosmic microwave background observations [10708-13]

$107080 G$ Metamaterial-based Toraldo pupils for super-resolution at millimetre wavelengths [10708-14]

SUBMM/FIR CAMERAS

$10708 \mathrm{JJ} \quad$ Optical design of the TolTEC millimeter-wave camera [10708-17]

10708 OK The SAFARI detector system [10708-18]

$10708 \mathrm{OL}$ Preflight characterization of the BLAST-TNG receiver and detector arrays [10708-19]

10708 OM MUSCAT: the Mexico-UK Sub-Millimetre Camera for AsTronomy [10708-20] 


\section{COHERENT DETECTION I}

10708 OY Evaluation of controllers for tuning digitizers in the ALMA interferometer [10708-32]

$10708 \mathrm{OZ} \quad \mathbf{4} \times 2 \mathrm{HEB}$ receiver at $4.7 \mathrm{THz}$ for GUSTO [10708-33]

1070812 Technical achievements of the ALMA future receiver development program at the National Astronomical Observatory of Japan [10708-36]

\section{COHERENT DETECTION II}

1070813 A proposal of a photonic local system for the extended Atacama large millimeter/submillimeter array and advanced radio interferometers [10708-37]

1070814 Planar superconductor-insulator-superconductor mixer array receivers for wide field of view astronomical observation [10708-38]

1070815 GLT receiver commissioning at JCMT and future JCMT instrumentation [10708-39]

1070816 Electronics instrumentation for the Greenland telescope [10708-40]

MULTIPLEXED READOUT

10708 1D Digital frequency multiplexing with sub-Kelvin SQUIDs [10708-47]

CMB INSTRUMENTS II

$107081 G$ The STRIP instrument of the Large Scale Polarization Explorer: microwave eyes to map the Galactic polarized foregrounds [10708-50]

SUBMM/FIR SPECTROMETERS II

1070810 The design and characterization of a $\mathbf{3 0 0}$ channel, optimized full-band millimeter filterbank for science with SuperSpec [10708-58]

\section{NEW DEVELOPMENTS}

$107081 \mathrm{U}$ Prime-Cam: a first-light instrument for the CCAT-prime telescope [10708-64]

iv 
$107081 \mathrm{~V}$ Development of a robust, efficient process to produce scalable, superconducting kilopixel farIR detector arrays [10708-65]

10708 IW Ultra-low-noise transition edge sensors for far infrared wavelengths: optical design, measurement and stray light control [10708-66]

10708 1X Eliminating stray radiation inside large area imaging arrays [10708-67]

POSTER SESSION: CMB DETECTORS I

$107081 Y \quad$ Design and characterization of the Cosmology Large Angular Scale Surveyor (CLASS) 93 GHz focal plane [10708-68]

POSTER SESSION: CMB INSTRUMENTS I

1070827 Cooldown strategies and transient thermal simulations for the Simons Observatory [10708-77]

1070828 The Cosmology Large Angular Scale Surveyor receiver design [10708-78]

1070829 Simons Observatory large aperture telescope receiver design overview [10708-79]

10708 2A Design and characterization of a ground-based absolute polarization calibrator for use with polarization sensitive CMB experiments [10708-80]

10708 2B QUBIC: the Q and U bolometric interferometer for cosmology [10708-81]

10708 2D BICEP array cryostat and mount design [10708-83]

$107082 \mathrm{E} \quad$ High-precision scanning water vapor radiometers for cosmic microwave background site characterization and comparison [10708-84]

10708 2F Preliminary scanning strategy analysis for the LSPE-STRIP instrument [10708-85]

POSTER SESSION: OPTICS I

10708 2G Design and performance of wide-band corrugated walls for the BICEP Array detector modules at $30 / 40 \mathrm{GHz}$ [10708-86]

$107082 \mathrm{H} \quad$ Next generation sub-millimetre wave focal plane array coupling concepts: an ESA TRP project to develop multichroic focal plane pixels for future CMB polarisation experiments [10708-87]

$1070821 \quad$ Simulations and performance of the QUBIC optical beam combiner [10708-88] 
10708 2K Ultra-thin large-aperture vacuum windows for millimeter wavelengths receivers [10708-90]

\section{Part Two}

10708 2M Variable-delay polarization modulators for the CLASS telescopes [10708-92]

10708 2N 2017 upgrade and performance of BICEP3: a 95GHz refracting telescope for degree-sCale CMB polarization [10708-93]

10708 2P SiAl alloy feedhorn arrays: material properties, feedhorn design, and astrophysical applications [10708-146]

\section{POSTER SESSION: COHERENT DETECTION}

$107082 R \quad$ Analysis techniques for complex field radiation pattern measurements [10708-96]

$1070825 \quad$ A VLBI receiving system for the South Pole Telescope [10708-97]

$107082 \mathrm{U} \quad$ Low-power CMOS digital electronics for radio, mm-wave and sub-mm astrophysics [10708-99]

10708 2V The new heterodyne receiver system for the ASTE radio telescope: three-cartridge cryostat with two cartridge-type superconducting receivers [10708-100]

10708 2X Development of a low-power cryogenic MMIC HEMT amplifier for heterodyne array receiver application [10708-102]

1070832 Q-band single pixel receiver development for the ngVLA and NRC [10708-36]

1070833 Performance of pre-production band 1 receiver for the Atacama Large Millimeter/submillimeter Array (ALMA) [10708-46]

1070834 A digital beamformer for the advanced focal array demonstrator (AFAD) [10708-58]

1070835 The first-light receivers for the Greenland Telescope [10708-149]

1070836 Progress in the construction and testing of the Tianlai radio interferometers [10708-150]

1070837 Overview of the East Asia ALMA development program [10708-152]

POSTER SESSION: FIR CAMERAS

1070838 Latest results and prospects of the ArTeMiS camera on APEX [10708-107] 
$1070839 \quad$ Upgrading SCUBA-2 with a newly designed thermal filter stack [10708-108]

POSTER SESSION: FIR DETECTORS

10708 3C Revisiting the optimization of the SCUBA-2 TES arrays for POL-2 and FTS-2 operations [10708-111]

POSTER SESSION: FIR SPECTROSCOPY

10708 3F TIME millimeter wave grating spectrometer [10708-114]

1070836 The optical design of a far Infrared spectrometer for SPICA: grating modules evaluation [10708-115]

POSTER SESSION: OPTICS II

$107083 \mathrm{H} \quad$ Use of evolutionary computing algorithms in the design of millimetre-wave metamaterial devices [10708-116]

$107083 \mathrm{I} \quad$ Fabrication and characterization of a NIR-FIR dichroic for the infrared interferometer BETTII [10708-117]

$107083 \mathrm{M}$ Characterizing and reducing the POL-2 instrumental polarization [10708-121]

POSTER SESSION: CMB DETECTORS II

1070830 Fabrication and characterization of cooled silicon bolometers for mm wave detection [10708-123]

POSTER SESSION: CMB INSTRUMENTS II

$107083 \mathrm{U} \quad$ Systematic error cancellation for the PIXIE four-port interferometric polarimeter [10708-129]

10708 3V Thermal architecture for the QUBIC cryogenic receiver [10708-130]

10708 3W Design and characterization of the POLARBEAR-2b and POLARBEAR-2c cosmic microwave background cryogenic receivers [10708-131]

$107083 X \quad$ Simons Observatory large aperture receiver simulation overview [10708-132] 
$107083 Z$ Studies of systematic uncertainties for Simons Observatory: detector array effects [10708-134]

1070840 Development of calibration strategies for the Simons Observatory [10708-135]

1070841 Designs for next generation CMB survey strategies from Chile [10708-136]

$1070842 \quad$ BoloCalc: a sensitivity calculator for the design of Simons Observatory [10708-137]

$1070843 \quad$ Broadband anti-reflective coatings for cosmic microwave background experiments [10708-138]

POSTER SESSION: MULTIPLEXING

1070844 The FDM readout for the LSPE/SWIPE TES bolometers [10708-139]

1070845 Performance of NbSi transition-edge sensors readout with a 128 MUX factor for the QUBIC experiment [10708-140]

$1070846 \quad$ Investigation of magnetic shielding for superconducting readout [10708-141]

POSTER SESSION: OPTICS III

1070847 Prototype design and evaluation of the nine-layer achromatic half-wave plate for the LiteBIRD low frequency telescope [10708-142]

1070849 Cross-polarization systematics due to Mizuguchi-Dragone condition breaking by a continuously rotating half-wave plate at prime focus in the Huan Tran telescope [10708-144]

10708 4A Multi-octave anti-reflection coating for polypropylene-based quasi-optical devices [10708-145]

10708 4B Feedhorn development and scalability for Simons Observatory and beyond [10708-147] 


\section{Authors}

Numbers in the index correspond to the last two digits of the seven-digit citation identifier (CID) article numbering system used in Proceedings of SPIE. The first five digits reflect the volume number. Base 36 numbering is employed for the last two digits and indicates the order of articles within the volume. Numbers start with 00, 01, 02, 03, 04, 05, 06, 07, 08, 09, OA, OB...0Z, followed by 10-1Z, 20-2Z, etc.

Adami, O.-A., 30

Addamo, Giuseppe, $1 \mathrm{G}$

Ade, Peter A. R., 05, 06, 07, OD, OK, OL, OM, 1W,

$2 \mathrm{~B}, 2 \mathrm{D}, 2 \mathrm{G}, 2 \mathrm{I}, 2 \mathrm{~K}, 2 \mathrm{~N}, 39,3 \mathrm{I}, 3 \mathrm{~V}, 43,45,4 \mathrm{~A}$

Agnese, P., 30

Ahmed, Z., 07, 1U, 2D, 2G, 2K, 2N, 43

Aikin, R. W., 07, 2D, 2G, 2K, 2N

Alexander, K. D., 07, 2D, 2G, 2K, 2N

Ali, Aamir M., 1U, 1Y, 27, 28, 29, 2M, 2P, 3X, 3Z, 40, $41,42,4 \mathrm{~B}$

Aliane, A., 30

Amico, G., 2B, 2I, 3V, 45

Anderson, A. J., 43

Anderson, Christopher J., 36

André, Ph., 38

Angile, Francisco E., OL

Ansari, Reza, 36

Appel, John W., 1Y, 28, 2M, 2P

Arnaud, A., 38

Arnold, Kam S., 29, 2A, 3W, 3X, 42, 49

Arrazola, David, 3G

Asada, Keiichi, 15, 16, 35

Asayama, Shin'ichiro, 12, 14, 2V, 33, 37

Ashton, Peter C., OL, 29, 3X, 42

Audley, Michael D., OK

Auguste, D., 2B, 2l, 3V, 45

Augusto Nunes Valle, Deniz, 1Y, 28, 2M

Aumont, J., 2B, 2I, 3V, 45

Austermann, Jason E., OJ, OL, 3Z, 4B

Avva, J. S., 43

Baker, L. A., 32

Baldini, A. M., 44

Banfi, S., 2B, 2I, 3V, 45

Barbarán, G., 2B, 2I, 3V, 45

Barkats, Denis, 07, 2D, 2E, 2G, 2K, 2N

Barron, Darcy, 42

Barry, Peter S., OM, 10, 1X

Baryshev, Andrey M., 1X, 2R

Baselmans, Jochem J. A., $1 X$

Basu Thakur, R., 43

Basu, K., $1 \mathrm{U}$

Battaglia, N., IU

Battaglia, Paola M., 1G, 2B, 2I, 3V, 45

Battistelli, E., 2B, 2I, 3V, 45

Baù, Alessandro, 1G, 2B, 2I, 3V, 45

Beall, James A., 4B

Beaudoin, Christopher, 2S

Becker, S., 30

Belenguer, Tomás, $3 G$
Bélier, B., 2B, 2I, 3V, 45

Bell, Graham S., 3C

Bender, Amy N., 1D, 43, 46

Benetti, Marco, 2F

Benford, Dominic, 06

Bennett, Charles L., 06, OF, 1Y, 28, 2M, 2P

Bennett, D., 2B, 2I, 3V, 45

Benson, B. A., 43

Benton, S. J., 07, 2D, 2G, 2K, 2N

Bergé, L., 2B, 2I, 3V, 45

Berkeley, Matthew, 2P

Bernard, J.-Ph., 2B, 2I, 3V, 45

Berry, David, 3M

Bersanelli, Marco, 1G, 2B, 2F, 2I, 3V, 45

Berthold, Ryan, 15

Bertoldi, F., IU

Bigot-Sazy, M.-A., 2B, 2I, 3V, 45

Bintley, Daniel, 15, 16, 35, 39, 3C, 3M

Bischoff, C. A., 07, 2D, 2G, 2K, 2N

Bleurvacq, N., 2B, 2I, 3V, 45

Bock, James, 07, 2D, 2G, 2K, 2N, 3F

Boenish, $\mathrm{H}_{\text {., }} 2 \mathrm{~N}$

Boettger, David, 49

Bolli, Pietro, $0 G$

Bonaparte, J., 2B, 2I, 3V, 45

Bond, J. Richard, 05, $1 \mathrm{U}$

Bonis, J., 2B, 2I, 3V, 45

Bordier, G., 2B, 2I, 3V, 45

Borrill, Julian, 41

Boulanger, François, 05

Bounissou, S., 30

Bowens-Rubin, R., 07, 2D, 2E, 2G, 2K, 2N

Bower, Geoff, 15

Bradford, Charles M., OK, 10, 3F

Bréelle, E., 2B, 2I, 3V, 45

Brevik, J. A., 07, 2D, 2G, 2K, 2N

Brewer, Michael, 1Y, 28, 2M

Brien, Thomas L. R., OM

Brown, Ari, IV

Brown, Michael L., 3Z, 41

Bruijn, Marcel P., OK

Bruno, Sarah Marie M., 42, 4B

Bryan, Sean A., 05, 0J, 40, 42

Bucher, M., 2H

Buder, I., 07, 2D, 2G, 2K, 2N

Bueno, Juan, $1 \mathrm{X}$

Bulcha, Berhanu, 2P

Bullock, E., 07, 2D, 2G, 2K, 2N

Bunn, E., 2B, 2I, 3V, 45 
Burgess, Tom, 34

Burke, D., 2B, 2I, 3V, 45

Bustos, Ricardo, 1U, 1Y, 28, 2M

Buza, V., 07, 2D, 2G, 2K, 2N

Buzi, D., 2B, 2I, 3V, 45

Buzzelli, A., 2B, 2l, 3V, 45

Caccianiga, Barbara, $1 \mathrm{G}$

Campagne, Jean-Eric, 36

Caprioli, Silvia, $1 \mathrm{G}$

Carlstrom, John E., 2S, 43

Carter, F. W., 43

Castillo-Domínguez, Edgar, $\mathrm{OM}$

Cavaliere, Francesco, 1G, 2B, 2I, 3V, 45

Cecil, T. W., 43

Cei, F., 44

Chan, Manwei, 1Y, 2M

Chang, C. L., 43, 46

Chang, Chih-Cheng, 33, 35

Chang, Shu-Hao, 16, 35

Chang, Song-Chu, 16

Chang, Tsu, 33

Chang, Tzu-Ching, $3 \mathrm{~F}$

Chanial, P., 2B, 2I, 3V, 45

Chapman, S. C., $1 \mathrm{U}$

Chapron, C., 2B, 2I, 3V, 45

Charlassier, R., 2B, 2I, 3V, 45

Charlet, Daniel, 36

Che, G., 10

Chen, Chung-Cheng, 16, 35

Chen, J., IW

Chen, Ming-Tang, 15, 16, 35

Chen, Xuelei, 36

Chen, Y., $2 X$

Chen, Zhiping, 36

Cheng, Jen-Chieh, 3F

Chiang, Po-Han, 33

Chien, Chen, 33

Chilson, Ryan, 16, 35

Chinone, Yuji, 3Z, 40, 41, 42, 49

Chiong, Chau-Ching, 33

Choi, Steve K., 4B

Chu, You-Hua, 33

Chung, D., $1 \mathrm{U}$

Chuss, David T., 06, 1Y, 28, 2M

Chuter, Tim, 15

Cianciara, Aleksander J., 36

Clay, W. H., 2E

Cleary, Joseph, 1Y, 28, 2M

Cleary, Kieran A., $1 \mathrm{G}$

Clerc, L., 38

Cliche, J. F., 43

Colazo, Felipe, 1 Y

Colom, Pierre, 36

Columbro, F., 2B, 2I, 3V, 45

Cong, Yanping, 36

Connors, J., 07, 2D, 2G, 2K, 2N

Cookson, Jamie L., 39, 3C

Coppi, Gabriele, 1U, 27, 29, 2B, 2I, 3V, 3W, 3X, 42, 45

Coppolecchia, A., 2B, 2I, 3V, 45
Corbett, Lance, OF

Cornelison, J., 07, 2D, 2G, 2K, 2N

Cothard, N. F., $1 \mathrm{U}$

Couchot, F., 2B, 2I, 3V, 45

Couto, Jullianna, $1 Y, 28,2 \mathrm{M}$

Crill, B. P., 07, 2D, 2G, 2K, 2N

Crites, Abigail, 3F

Crowley, K. D., 2A

Crowley, Kevin T., 3Z, 40, 42, 4B

Crumrine, Michael, 07, 2D, 2G, 2K, 2N

Cukierman, Ari, 3Z, 42, 43

Culp, T., 2E

Cursey, Paul W., 06

Cuttaia, Francesco, $1 \mathrm{G}$

D'Agostino, Francesco, OG

D'Agostino, R., 2B, 2l, 3V, 45

Dahal, Sumit, 1Y, 28, 2M, 2P

D'Alessandro, G., 2B, 2I, 3V, 45

Das, Santanu, 36

Datta, Rahul, 06

Davis, Kristina, $2 R$

de Bernardis, P., 2B, 2H, 2I, 3V, 45

de Breuck, C., 38

De Gasperis, G., 2B, 2I, 3V , 45

DeGeorge, Martin, $2 M$

de Haan, T., 43

Delabrouille, J., $2 \mathrm{H}$

de Lange, Gert, OK, $3 \mathrm{G}$

De Leo, M., 2B, 2I, 3V, 45

Delisle, C., 38

Del Torto, Francesco, $1 \mathrm{G}$

Dempsey, Jessica, 15, 16, 35, 3M

Denis, Kevin L., 1Y, 28, 2M, 2P

Deo, P., 2H

De Petris, M., 2B, 2H, 2I, 3V, 45

Devlin, Mark J., 05, 0L, 27, 29, 3X

Dhabal, Arnab, 31

Dicker, Simon R., 1U, 27, 29, 3X, 42, 4B

Di Donato, A., 2B, 2I, 3V, 45

Dierickx, Marion I., 07, 2D, 2G, 2K, 2N

Ding, J., 43

Dobbs, Matthew A., 1D, 43

Dober, Bradley, OL, 3Z, 4B

Doeleman, Shepherd S., 16, 2 S

Doherty, S., 2H

Dotson, Jessie L., 06

Doumayrou, E., 38

Doyle, Simon M., 05, 0M, $1 X$

Duband, L., 07, 2D, 2G, 2K, 2N, 38

Dubreuil, D., 38

Duell, C. J., $1 \mathrm{U}$

Duff, Shannon M., 1U, 3Z, 4B

Dumaye, L., 38

Dumoulin, L., 2B, 2I, 3V, 45

Dunkley, Jo, 3Z, 42

Dünner, Rolando, 1Y, 28, 2M

Dussopt, L., 30

Dutcher, D., 43

Dvorkin, C., 07, 2D, 2G, 2K, 2N

Effland, John, 33 
Eggens, Martin, $3 G$

Eimer, Joseph R., 06, 1Y, 28, 2M

Ercolani, E., 38

Erler, J., $1 \mathrm{U}$

Errard, Josquin, $3 Z$

Essinger-Hileman, Thomas M., 06, OF, 1Y, 28, 2M, $2 \mathrm{P}$

Etchegoyen, A., 2B, 2I, 3V, 45

Everett, W., 43

Evers, Jaap, $3 G$

Ezaki, S., 14

Fabbian, Giulio, 3Z, 40, 42

Fafone, Viviana, $1 \mathrm{G}$

Farooqui, Zunnoorain, $1 G$

Fasciszewski, A., 2B, 2I, 3V, 45

Fernández, Marianela, $3 G$

Ferrari, Lorenza, 1X

Ferrusca, Daniel, OJ, OM

Fich, M., $1 \mathrm{U}$

Filippini, Jeffrey, 05, 07, 2D, 2G, 2K, 2N

Finger, Ricardo, 33

Fissel, Laura, 05

Fixsen, Dale J., 06, 31, 3U

Fliescher, S., 07, 2D, 2G, 2K, 2N

Fluxa, Pedro, 1Y, 28, 2M

Folkers, Thomas W., $2 S$

Forbes, David, 2S

Foster, A., 43

Franceschet, Cristian, 1G, 2B, 2F, 2I, 3V, 45

Friberg, Per, 15, 16, 35, 3C, 3M

Fu, J., 43

Fu, Kuo-Chieh, 16

Fujii, Yasunori, 2V

Gaier, Todd C., $1 G$

Galitzki, Nicholas, OL, 1U, 27, 29, 2A, 3X, 42

Gallais, P., 38

Gallardo, Patricio A., 1U, 27, 29, 3X, 3Z, 40, 41, 42

Galli, L., 44

Gallichio, J., 43

Gamboa Lerena, M. M., 2B, 2I, 3V, 45

Gandilo, Natalie N., 06

Gao, Jian-Rong, OK, $\mathrm{OZ}$

Gao, Jiansong, OL

García, B., 2B, 2l, 3V, 45

Garrido, X., 2B, 2I, 3V, 45

Gascard, Thomas, $0 M$

Gaspard, M., 2B, 2I, 3V, 45

Gault, A., 2B, 2I, 3V, 45

Gayer, D., 2B, 2I, 3V, 45

Gayley, Kevin G., 36

Geng, Jingchao, 36

Génova Santos, Ricardo T., IG

Gerbino, Martina, 40

Gervasi, Massimo, 1G, 2B, 2I, 3V, 45

Ghigna, Tommaso, OE, $1 \mathrm{G}$

Ghribi, A., 2H

Giard, M., 2B, 2I, 3V, 45

Gilbert, Adam J., 1D, 43

Giraud-Héraud, Y., 2B, 21, 3V, 45

Glenn, J., 10
Goeckner-Wald, Neil, 3Z, 40, 41

Goldie, D. J., IW

Goldsmith (PI), Paul F., 2U

Golec, Joseph E., 4B

Gómez Berisso, M., 2B, 2I, 3V, 45

Gómez, Victor, OM

González Fernández, Luis Miguel, 3G

Gonzalez, Alvaro, 12, 33, 37

González, M., 2B, 2I, 3V, 45

Gordon, Sam, OL

Goudon, V., 30

Gradziel, M., 2B, 2H, 2I, 3V, 45

Grandsire, L., 2B, 2I, 3V, 45

Grassi, M., 44

Graves, Sarah, 3M

Grayson, J. A., 07, 2D, 2G, 2K, 2N

Greer, Christopher H., $2 S$

Groh, J. C., 3W, 43

Groppi, Christopher E., 05, OL, 0Z, 2R, 2U

Grunberg, Theodore W., 2M

Gudmundsson, Jon E., 42

Guerrard, E., 2B, 2I, 3V, 45

Guns, S. T., 43

Guo, Haiquan, OF

Guo, X., 3W

Guyser, R., 43

Hailey-Dunsheath, S., 10

Hall, G., 07, 2D, 2G, 2K, 2N

Halpern, Mark, 06, 07, 1Y, 28, 2D, 2G, 2K, 2M, 2N

Halverson, N. W., 43

Hamilton, J.-Ch., 2B, 2I, 3V, 45

Han, Chih-Chiang, 15, 16, 35

Han, Kou-Chang, 16

Hao, Jie, 36

Harari, D., 2B, 2I, 3V , 45

Hargrave, Peter C., OM

Harke-Hosemann, A. H., 43, 46

Harrington, Kathleen, $1 Y, 28,2 M$

Harrington, N. L., 43

Harrison, S. A., 07, 2D, 2G, 2K, 2N

Harrison, Stephen T., 34

Hasegawa, Yutaka, 16, 35

Haynes, V., 2B, 2I, 3V, 45

Hazumi, Masashi, OE, 47, 49

Healy, Erin, $4 \mathrm{~B}$

Helson, Kyle, 1Y, 28

Henderson, Shawn W., 1U, 29

Henke, Doug, 32, 33

Henning, J. W., 43

Henrot-Versillé, S., 2B, 2l, 3V, 45

Herrera, Daniel, OY

Herter, T. L., $1 \mathrm{U}$

Hijmering, Richard A., OK

Hildebrandt, S. R., 07, 2D, 2G, 2K, 2N

Hill, Charles A., OE, 42, 4B

Hills, R., 2E

Hilton, Gene C., 06, 07, 0L, 1U, 1V, 1Y, 28, 2D, 2G,

$2 \mathrm{~K}, 2 \mathrm{M}, 2 \mathrm{~N}$

Hinshaw, Gary F., 06, 1Y, 28, 2M

Ho, Chin-Ting, 33 
Ho, Paul T. P., 15, 16, 33, 35

Ho, Shuay-Pwu Patty, 29, 3Z, 4B

Hoang, D. T., 2B, 2I, 3V, 45

Hoh, Jonathan R., $2 \mathrm{U}$

Holder, Gilbert, 05

Holzapfel, W. L., 43

Hornsby, Amber L., OM

Hovey, Gary J., 34

Howe, L., 3W

Hsieh, Fang-Chia, 33

Hsu, Sung-Po, 33

$\mathrm{H} \cup, \mathrm{Q}_{\text {., }} \mathrm{OZ}$

Huang, Chih-Wei, 15, 16

Huang, Ji-Dian, 33, 35

Huang, N., 43

Huang, Qizhi, 36

Huang, Yau-De (Ted), 16, 33, 35

Hubmayr, Johannes, 05, 0L, 1U, 1Y, 28, 29, 2M, 3Z, 42, 4B

Hughes, David, OM

Hui, Howard, 07, 2D, 2G, 2K, 2N

Hunacek, Jonathon, 3F

Hwang, Yuh-Jing, 33

Iguchi, Satoru, 12, 14, 33, 37

lida, Teruhito, OE

Imada, Hiroaki, $\mathrm{OE}, 47$

Inata, Motoko, 2V

Incardona, Federico, 1G, 2B, 2F, 2I, 3V, 45

Inove, Makoto, 16, 35

Iono, Daisuke, 12, 2V, 33, 37

lovenitti, Simone, $1 \mathrm{G}$

Irwin, Kent, 06, 07, 1U, 1V, 2D, 2G, 2K, 2N, 43

Ishino, Hirokazu, OE, 47

Ito, Tetsuya, 2V

Iuliano, Jeffrey, 1Y, 28, 2M

Izumi, Natsuko, 2V

Jackson, Brian D., OK

Jellema, Willem, 2R, $3 G$

Jeong, O. B., 43

Jhabvala, Christine A., 06

Jian, Shou-Ting, 33

Jiang, Homin, 16

Jiang, N., 32

Jonas, M., 43

Jones, A., 43

Jones, Mike, $1 G$

Jules, E., 2B, 2l, 3V, 45

Kamazaki, Takeshi, 2V

Kanai, Hiroaki, OE

Kang, Jae Hwan, 07, 2D, 2G, 2K, 2N

Kangaslahti, Pekka, $1 \mathrm{G}$

Kaplan, J., 2B, 2I, 3V, 45

Karakla, John, 1Y, 28, 2M

Karkare, K. S., 07, 1O, 2D, 2G, 2K, 2N

Karpel, E., 07, 2D, 2G, 2K, 2N

Kasica, Richard, 31

Katayama, Nobuhiko, OE, 47

Kataza, Hirokazu, OE

Kaufman, J. P., 07, 2A, 2D, 2G, 2K, 2N

Kaya, H., $3 \mathrm{O}$
Keating, Brian, 07, 27, 29, 2A, 2D, 2G, 2K, 2N, 3W, $3 X, 3 Z, 40,42,49$

Kefeli, S., 07, 2D, 2G, 2K, 2N

Keith, Celeste S., 36

Kemper, Ciska, 33

Kernasovskiy, S. A., 07, 2D, 2G, 2K, 2N

Keskitalo, Reijo, 41

Khaire, T. S., 43

Khalatpour, A., $\mathrm{OZ}$

Kim, Junhan, $2 S$

Kimball, Mark, 06

Kimura, Kimihiro, 16, 35

Kiuchi, H., 13, 37

Klein, Jeffrey, OL

Knee, L. B. G., 32

Koch, Patrick Michel, 16, 33

Kofman, A. M., 43

Kogut, Alan J., 06, $3 U$

Kojima, T., 12, 2X, 37

Komatsu, Kunimoto, OE, 47

Konishi, Kuniaki, OE

Koopman, Brian, 1U, 40

Korman, M., 43

Korotkov, A., 2B, 2I, 3V, 45

Kosugi, G., 37

Kovac, John M., 07, 2D, 2E, 2G, 2K, 2N

Koyama, Shoko, 15, 16

Kristukat, C., 2B, 2I, 3V, 45

Kroug, M., 12, 37

Kubik, D. L., 43

Kubo, Derek, 15, 16, 35

Kuhlmann, S., 43

Kulesa, C., $0 Z$

Kuo, C.-L., 07, 2D, 2G, 2K, 2N, 43

Kusaka, Akito, OE, 3Z, 40, 41, 42, 49

Kuzmin, L., 2H

Laauwen, W., $\mathrm{OZ}$

Lamagna, L., 2B, 2I, 3V, 45

Larsen, N. A., 07, 2D, 2E, 2G, 2K, 2N

LaU, K., 07, 2D, 2G, 2K, 2N

Lauria, Eugene F., $2 S$

Leduc, H. G., 10

Lee, Adrian T., 29, 3W, 3X, 41, 42, 43, 49

Leitch, E. M., 07, 2D, 2G, 2K, 2N

Le-Pennec, Y., 38

$\mathrm{Li}, \mathrm{Chao}, 36$

Li, Chao-Te, 35, 3F

$\mathrm{Li}$, Dale, $\mathrm{OL}$

Li, Jixia, 36

Li, Shaoliang, 39, 3C

$\mathrm{Li}$, Yaqiong, 4B

Li, Yichao, 36

Li, Z., $2 X$

Limon, Michele, 27, 29, $3 x$

Lin, Lupin, 15

Liv, Chao, 36

Liv, Ching-Tang, 16, 33, 35

Liu, J., 14, $2 X$

Liu, Kuan-Yu, 15, 16, 35

Liu, Tao, 36 
Ljusic, Zoran, 34

Llombart, Nuria, $1 \mathrm{X}$

Locke, L., 32

Lortholary, M., 38

Loucatos, S., 2B, 2I, 3V , 45

Lovis, T., 2B, 2l, 3V, 45

Lourie, Nathan P., OL

Lowe, lan, OL

Lowe, Luke, 06

Lowitz, Amy E., 1D, 2B, 2I, 3V, 43, 45

Lowry, L., 3W

Lu, Li-Ming, 16

Lueker, M. V., 07, 2D, 2G, 2K, 2N

Lukovic, V., 2B, 2I, 3V, 45

Lungu, Marius, 27, 29, 3X, 4B

Luterstein, R., 2B, 2I, 3V, 45

Maestre, S., 45

Maffei, Bruno, 0G, 2B, 2H, 2I, 3V, 45

Magneville, Christophe, 36

Maher, Stephen, IV

Mainini, Roberto, $1 G$

Maino, Davide, 1G, 2F

Mak, Suet Ying D., 41

Maki, Muneyoshi, OE

Mani, Hamdi, OL

Maris, Michele, IG

Marnieros, S., 2B, 2I, 3V, 45

Marriage, Tobias, OF, 1Y, 28, 2M, 2P

Marriner, John P., 36

Marrone, Daniel P., 2S

Martignac, J., 38

Martin, Jean-Michel, 36

Martin-Cocher, Pierre, 16

Marty, W., 45

Masi, S., 2B, 2H, 2I, 3V, 45

Massingill, Kyle D., $2 S$

Matsuda, Frederick T., 40, 42, 49

Matsumura, Tomotake, OE, 47

Matsushita, Satoki, 15, 16, 35

Mattei, A., 2B, 2I, 3V, 45

Mauskopf, Philip D., 05, 0J, OL, 10, 29, 40, 42

May, Andrew J., 27, 29, 2B, 2I, 3V, 3W, 3X, 45

Mayer, Evan, 2S

McCallum, Nialh, 3Z, 41

McCulloch, M. A., 2B, 21, 3V, 45

McGeehan, R., 10

McGregor, Helen, 15

McKenney, Christopher M., OL

McMahon, Jeffrey John, 05, 06, 0J, 1U, 1Y, 27, 28,

$29,2 \mathrm{M}, 3 \mathrm{X}, 3 \mathrm{Z}, 40,41,42,4 \mathrm{~B}$

Meador, Mary Ann B., OF

Medina, M. C., 2B, 2I, 3V, 45

Megerian, K. G., 07, 2D, 2G, 2K, 2N

Mele, L., 2B, 2I, 3V, 45

Melhuish, S., 2B, 2I, 3V, 45

Mena, Patricio, $1 \mathrm{G}$

Mennella, Aniello, 1G, 2B, 2F, 2I, 3V, 45

Mentzell, John E., 3l

Meyer, S. S., 43

Michalik, D., 43
Migliozzi, Massimo, OG

Miller, Nathan J., 1Y, 28, 2M

Miller, Timothy M., 06

Mirel, Paul, 06

Mirzaei, B., $\mathrm{OZ}$

Molina, Rocío, $1 \mathrm{G}$

Moncelsi, L., 07, 2D, 2G, 2K, 2N

Moniez, Marc, 36

Montaña, Alfredo, 0J

Montgomery, J., 43

Montier, L., 2B, 2I, 3V, 45

Morata, Oscar, 33

More, N., OZ

Morgante, Gianluca, $1 G$

Moseley, Paul, OD

Moseley, Samuel H., 06, $1 \mathrm{~V}$

Mugnai, Daniela, OG

Mundo, L. M., 2B, 2I, 3V, 45

Murphy, J. A., 2B, 2H, 2I, 3V, 45

Murphy, J. D., 2B, 2I, 3V, 45

Murray, N., $1 \mathrm{U}$

Murugesan, Vignesh, $1 \mathrm{X}$

Nadolski, A., 43

Nagy, Johanna, 05

Najarro, Francisco, $3 \mathrm{G}$

Nakamura, Shogo, OE

Namikawa, T., 07, 2D, 2G, 2K, 2N

Nati, Federico, OL, 3Z, 40

Natoli, T., 43

Navaroli, M. F., 2A

Navarrini, Alessandro, $0 G$

Netterfield, C. Barth, 05, 07, 2D, 2G, 2N

$\mathrm{Ng}$, Richard, 4A

Nguyen, Chi H., $2 \mathrm{~S}$

Nguyen, H. T., 07, 2D, 2G, 2K, 2N

Nguyen, H., 43

Nicolò, D., 44

Niemack, Michael D., 05, 1U, 27, 29, 3X, 3Z, 41, 42

Nikola, T., $1 \mathrm{U}$

Nishioka, Hiroaki, 15, 16

Noble, G. I., 43

Noguchi, T., 12, 14

Nolta, M., $1 \mathrm{U}$

Norton, Timothy J., 16, 35

Novak, Giles, 05, 0J, OL

Noviello, F., $2 \mathrm{H}$

Novosad, V., 43, 46

Nuñez, Carolina, 1Y, 28, 2M

O'Brient, R., 07, 2D, 2G, 2K, 2N

Ogawa, Hideo, 16, 35

Ogburn, R. W., IV, 07, 2D, 2G, 2K, 2N

Ohsaki, Hiroyuki, OE

Okuda, Takeshi, 2V

Olivieri, E., 2B, 2I, 3V, 45

Olmi, Luca, OG

Orfei, Alessandro, 0G

Orlowski-Scherer, John L., 1U, 27, 29, 3X, 4B

Oshiro, Peter, 15, 16

O'Sullivan, C., 2B, 2H, 2I, 3V, 45

Ouvrier-Buffet, J.-L., 30 
Oxholm, Trevor M., 36

Padilla, Ivan L., 1Y, 28, 2M

Padin, S., 43

Pagano, L., $2 \mathrm{H}$

Page, Lyman, 41

Paiella, A., 2B, 2I, 3V, 45

Paine, S., 2E

Pajot, F., 2B, 2I, 3V, 45

Palladino, S., 07, 2D, 2G, 2K, 2N

Palma, Gonzalo, 1Y, 28, 2M

Pan, Z., 43

Parker, Lucas, 1Y, 28, 2M

Parrianen, Josie D. A., OM

Parshley, S. C., IU

Parsons, Harriet, $3 \mathrm{M}$

Pascale, Enzo, 05, OL, OM

Passerini, Andrea, 1G, 2B, 2I, 3V, 45

Pastoriza, H., 2B, 2I, 3V, 45

Patel, Nimesh A., 16

Pawlyk, Samuel, 06

Pearson, J., 43

Pelosi, A., 2B, 2I, 3V, 45

Pen, Ue-li, 36

Pentacoff, Chris, $2 \mathrm{~K}$

Perbost, C., 2B, 2I, 3V, 45

Perdereau, Olivier, 2B, 2I, 36, 3V, 45

Perez, Abel, OM

Perez-de-Taoro, Maria del Rosario, IG

Peterson, Jeffrey B., 36

Petroff, Matthew, 1Y, 28, 2M

Peverini, Oscar A., $1 G$

Pezzotta, Federico, 1G, 2B, 2l, 3V, 45

Piacentini, F., 2B, 2H, 2l, 3V, 45

Piat, M., 2B, 2H, 2I, 3V, 45

Piccirillo, Lucio, 27, 29, 2B, 2I, 3V, 3W, 3X, 45

Piendibene, M., 44

Pincella, Claudio, $1 \mathrm{G}$

Pisano, Giampaolo, 05, OD, 0G, 0L, 2B, 2H, 2I, 3H, $3 \mathrm{~V}, 45,4 \mathrm{~A}$

Pocas, S., 30

Polakovic, T., 46

Polenta, G., 2B, 2I, 3V, 45

Posada, C. M., 43

Pospieszalski, Marian, 33

Pradenas Márquez, Bastian, 1Y, 28, 2M

Prêle, D., 2B, 2I, 3V, 45

Pryke, C., 07, 2D, 2G, 2K, 2N

Puddu, R., 2B, 2I, 3V, 45

Puglisi, Giuseppe, 27, 29, 3X, 3Z, 40, 41, 42

Quan, W., 43

Rabaud, W., 30

Racine, B., 07, 2D, 2G, 2K, 2N

Rafael, García, 3G

Raffin, Philippe, 16, 35

Rahlin, A., 43

Rambaud, D., 2B, 2I, 3V, 45

Rao, Ramprasad, 15

Realini, Sabrina, 1G, 2F

Reck, T., 10

Redford, J., 10
Reeves, Rodrigo, 1Y, 28, 2M

Reichardt, Christian L., 3Z, 40

Reiland, George, 25

Reintsema, Carl, 1Y, 28, 2M

Relland, J., 38

Reshetov, V., 32

Revéret, V., 38, 30

Reyes, Nicolás, 1G, 33

Rhoades, Gary, 28

Richter, S., 07, 2D, 2G, 2K, 2N

Ridder, Marcel L., OK

Riechers, D. A., $1 \mathrm{U}$

Riminesi, Cristiano, OG

Rinehart, Stephen A., 31

Ringegni, P., 2B, 2I, 3V, 45

Robinson, M., $2 \mathrm{H}$

Rocchi, Alessio, $1 G$

Rodriguez, L., 38, 30

Rodriguez, Samelys, 06

Roelfsema, Peter R., OK, 3G

Romero, G. E., 2B, 2I, 3V, 45

Rossi, K., $1 \mathrm{U}$

Rostem, Karwan, OF, 1V, 1Y, 28, 2M, 2P

Roussel, F., 38

Rowe, Sam, OM

Rubiño-Martín, José A., $1 \mathrm{G}$

Ruhl, John, 05, 43

Saez, Alejandro, OY

Sagliocca, Marco, 1 Y

Saini, Kamaljeet, 33

Sakurai, Haruyuki, OE

Sakurai, Yuki, OE, 47

Salatino, Maria, 27, 29, 2B, 21, 3V, 3X, 3Z, 40, 41, 42, 45, 4B

Sánchez-Argüelles, David, OJ

Sandri, Maura, $1 \mathrm{G}$

Sartor, Stefano, $1 \mathrm{G}$

Sathyanarayana Rao, Mayuri, 29, 3Z, 42

Satou, Naohisa, 2V

Sauvageot, J.-L., 30

Savini, Giorgio, OD, 3M

Sayre, J. T., 43

Schillaci, A., 07, 2B, 2D, 2G, 2I, 2K, 2N, 3V, 45

Schuller, F., 38

Schwarz, R., 07, 2D, 2G, 2K, 2N

Scóccola, C. G., 2B, 2I, 3V, 45

Scott, Douglas, 05, $1 \mathrm{U}$

Scully, S., 2B, 2I, 3V, 45

Shan, W., 12, 14, 2X, 37

Sharp, Elmer H., 06, $1 \mathrm{~V}$

Shaw, Yang-Tai, 16

Sheehy, C. D., 07, 2D, 2E, 2G, 2K, 2N

Shi, Huli, 36

Shimomura, Toshiki, OE

Shirokoff, E., 1O, 43

Shirron, Peter, 06

Shitvov, Alexey, OD, OG

Shu, Lin, 36

Sierra, Carlos, $1 \mathrm{U}, 42$

Signorelli, G., 44

xiv 
Siles, Jose V., $2 U$

Silva, J. R. G., OZ

Silva-Feaver, Max, 1U, 29, 3X, 37

Silverberg, Robert F., 31

Simon, Sara M., 0J, 1U, 27, 29, 3X, 3Z, 40, 42, 4B

Sinclair, Adrian, $\mathrm{OL}$

Siritanasak, Praween, $3 Z$

Smecher, G., 43

Sobrin, J. A., 43

Soler, Juan D., 05, OL

Soliman, A., 07, 2D, 2G, 2K, 2N

SooHoo, Jason, $2 \mathrm{~S}$

Soria, Mary, $1 G$

Spinella, F., 44

Spinelli, S., 2B, 2I, 3V, 45

Srikanth, Sivasankaran, 35

Srinivasan, Ranjani, 15, 16, 35

St. Germaine, T., 07, 2D, 2G, 2K, 2N

Stacey, G. J., $1 \mathrm{U}$

Staggs, Suzanne, 29, 3Z, 42, 4B

Staguhn, Johannes G., 06, $1 \mathrm{~V}$

Staniszewski, Z. K., 07, 2D, 2G, 2K, 2N

Stark, Antony A., 2S, 43

Stebbins, Albert, 36

Stebor, N., 3W

Steinbach, B., 07, 2D, 2G, 2K, 2N

Stevens, Jason R., 1U, 41

Stolpovskiy, M., 2B, 2I, 3V, 45

Stompor, R., $2 \mathrm{H}$

Story, K. T., 43

Suarez, F., 2B, 2I, 3V, 45

Sudiwala, Rashmikant, 07, 1W, 2D, 2G, 2K, 2N, 39

Sugai, Hajime, OE, 47

Sugiyama, Shinya, $0 \mathrm{E}$

Sullivan, Dan F., 06

Sun, Shijie, 36

Suzuki, Aritoki, 3Z, 42, 43

Suzuki, Junichi, OE

Switzer, Eric R., 06

Takaku, Ryota, OE, 47

Takaku, Ryota, 47

Takakura, Satoru, 49

Talvard, M., 38

Tang, Adrian J., $2 \mathrm{U}$

Tapia, Valeria, 1G, 33

Taraschi, Peter, 06

Tartari, A., 2B, 2H, 2I, 3V , 44, 45

Tashiro, Makoto, $\mathrm{OE}$

Teply, Grant, 07, 27, 2A, 2D, 2G, 2K, 2N, 3W, 3Z, $40,41,42$

Terada, Yukikatsu, OE

Terao, Yutaka, OE

Terenzi, Luca, $1 \mathrm{G}$

Thermeau, J.-P., 2B, 2I, 3V, 45

Thoen, David J., IX

Thomas, C. N., IW

Thomas, Daniel B., 3Z, 41

Thompson, Jonathan A., $3 \mathrm{H}$

Thompson, K. L., 07, 2D, 2G, 2K, 2N, 43

Thornton, Robert, 27, 29, 3X
Timbie, Peter T., 2B, 21, 36, 3V, 45

Tolan, J. E., 07, 2D, 2G, 2K, 2N

Tomasi, Maurizio, 1G, 2F

Tommasi, Elisabetta, $1 \mathrm{G}$

Torchinsky, Steve, 2B, 2I, 36, 3V, 45

Torrecillas, R., 30

Torres, Josefina, $3 G$

Traini, A., 2H

Trappe, Neal A., OK, 1W, 2H

Tristram, M., 2B, 2I, 3V, 45

Truongcanh, V., 2B, 2l, 3V, 45

Tsai, C., 3W

Tsay, Jinn jy, 33

Tseng, Yen-Hsiang, 33

Tucker, Carole E., 05, 06, 07, OD, OG, OL, OM, 2B

2D, 2G, 2I, 2K, 2N, 39, 3I, 3V, 43, 45, 4A

Tucker, Gregory S., 2B, 2I, 36, 3V, 45

Turner, A. D., 07, 2D, 2G, 2K, 2N

Uemizu, Kazunori, 2V

Ullom, Joel N., OL, 1U, 29, 3Z, 42

Umiltà, C., 07, 2D, 2G, 2K, 2N

Utsunomiya, Shin, OE

U-Yen, Kongpop, 1Y, 2P

Vaccaro, D., 44

Vanderlinde, K., 43

van der Vorst, M., $2 \mathrm{H}$

Van Engelhoven, Trevor, 1Y, 28, 2M

Vanneste, S., 2B, 2I, 3V, 45

Van Zyl, J., 2G

Vavagiakis, Eve M., 1U, 27, 29, 3X, 41

Veach, Todd, 31

Veidt, Bruce, 34

Ventura González, Salvador, OM

Vergès, Clara, $3 Z$

Vertatschitsch, Laura, 25

Vialle, C., 30

Vieira, Joaquin, 05, 43

Vieregg, A. G., 07, 2D, 2E, 2G, 2K, 2N

Viganó, Daniele M., 1G, 2B, 2I, 3V, 45

Villa, Fabrizio, $1 \mathrm{G}$

Virone, Giuseppe, $1 \mathrm{G}$

Vissers, Michael R., OL, 1U, $3 Z$

Visticot, F., 38

Vittorio, N., 2B, 2I, 3V, 45

Voisin, F., 2B, 2I, 3V, 45

Volpe, Angela, $1 G$

Walker, C., $\mathrm{OZ}$

Walker, I., IW

Walker, S., $1 \mathrm{U}$

Walther, Craig, 15, 16, 35

Walts, Alexander R., 06

Wandui, A., 07, 2D, 2G, 2K, 2N

Wang, Bingjie, $1 Y, 28,2 M$

Wang, Qinan, 1Y, 28, 2M

Wang, G., 43

Wang, Guisong, 36

Wang, Rongli, 36

Wang, Xin, 36

Wang, Yougang, 36

Watkins, Bob, $1 \mathrm{G}$ 
Watson, B., 2B, 2I, 3V, 45

Watts, Duncan, 1Y, 28, 2M

Weber, A. C., 07, 2D, 2G, 2K, 2N

Wei, Ta-Shun, 15, 16, 35, 3F

Weiland, Janet, 1Y, 28, 2M

Weintroub, Jonathan, $2 S$

Westbrook, Benjamin, 29, 3Z, 42

Wheeler, J., 10

Whitehorn, N., 43

Whitton, Jeremy D., $2 \mathrm{U}$

Wicek, F., 2B, 2I, 3V, 45

Wiebe, D. V., 07, 2D, 2G, 2K, 2N

Williams, E. A., IW

Williams, Paul A., OL

Willmert, J., 07, 2D, 2G, 2K, 2N

Wilson, Grant, 0J

Withington, Stafford, OK, IW

Wollack, Edward J., 06, 0F, 1U, 1V, 1Y, 28, 29, 2M,

2P, 3Z, 41, 4B

Wong, C. L., 07, 2D, 2G, 2K, 2N

Wu, Fengquan, 36

Wu, W. L. K., 07, 2D, 2G, 2K, 2N

$\mathrm{Xu}$, Yidong, 36

Xu, Zhilei, 1U, 1Y, 27, 28, 29, 2M, 3X, 3Z, 40, 41, 42,

$4 \mathrm{~B}$

Yamamoto, Ryo, OE

Yan, Zi'ang, 1Y, 28, 2M

Yang, Chia-Hsiang, 33

Yang, H., 07, 2D, 2G, 2K, 2N

Yates, Stephen J. C., 1X, 2R

Yefremenko, V., 43

Yen, Sheng-Feng, 16

Yeung, Keith, 33

Yoon, K. W., 07, 2D, 2G, 2K, 2N, 43

Young, A., $0 Z$

Young, André, $2 S$

Young, M. R., 43

Yu, Chen-Yu, 16

Yu, Kaifeng, 36

Yumoyo, Junji, OE

Yurduseven, Ozan, $1 \mathrm{X}$

Zacchei, Andrea, $1 \mathrm{G}$

Zannoni, Mario, 1G, 2B, 2I, 3V, 45

Zeng, Lingzhen, 1Y, 28, 2M, 2P

Zhang, Cheng, 07, 2D, 2G, 2K, 2N

Zhang, Jessie, 31

Zhang, Jiao, 36

Zhang, Juyong, 36

Zhang, Le, 36

Zhang, $X ., 2 X$

Zhu, C., $2 \mathrm{H}$

Zhu, Cheng, 4A

Zhu, Jialu, 36

Zhu, Ningfeng, 1U, 27, 29, 3X, 3Z, 40, 41, 42, 4B

Zmuidzinas, J., 10

Zullo, A., 2B, 2I, 3V, 45

Zuo, Shifan, 36 


\section{Conference Committee}

Symposium Chairs

Allison A. Barto, Ball Aerospace \& Technologies Corporation (United States)

Suzanne K. Ramsay, European Southern Observatory (Germany)

Symposium Chairs

Satoru Iguchi, National Astronomical Observatory of Japan (Japan)

Alison B. Peck, Gemini Observatory (United States)

Conference Chairs

Jonas Zmuidzinas, California Institute of Technology (United States)

Jian-Rong Gao, SRON Netherlands Institute for Space Research

i(Netherlands) and Technische Universiteit Delft (Netherlands)

Conference Program Committee

Masashi Hazumi, High Energy Accelerator Research Organization, KEK (Japan)

Kent D. Irwin, Stanford University (United States)

Karl Schuster, IRAM-Domaine Université de Grenoble (France)

Gordon J. Stacey, Cornell University (United States)

Neil A. Trappe, National University of Ireland, Maynooth (Ireland)

Carole E. Tucker, Cardiff University (United Kingdom)

Christopher K. Walker, The University of Arizona (United States)

Session Chairs

1 CMB Instruments I

Sunil R. Golwala, California Institute of Technology (United States)

2 CMB Detectors I

Masashi Hazumi, High Energy Accelerator Research Organization, KEK (Japan)

3 Optics I

Neal A. Trappe, National University of Ireland, Maynooth (Ireland)

4 Submm/FIR Cameras

Akira Endo, Technische Universiteit Delft (Netherlands) 
5 Submm/FIR Spectrometers I

Gordon J. Stacey, Cornell University (United States)

6 Submm/FIR Detectors I

Omid Noroozian, NASA Goddard Space Flight Center (United States)

7 Coherent Detection I

Christopher Grappi, The University of Arizona (United States)

8 Coherent Detection II

Jian-Rong Gao, SRON Netherlands Institute for Space Research

(Netherlands) and Technische Universiteit Delft (Netherlands)

9 Multiplexed Readout

Jonas Zmuidzinas, California Institute of Technology (United States)

$10 \mathrm{CMB}$ Instruments II

Johannes G. Staguhn, NASA Goddard Space Flight Center (United States)

11 CMB Detectors II

Jochem J. A. Baselmans, SRON Netherlands Institute for Space Research (Netherlands)

12 Submm/FIR Spectrometers II

Erik Shirokoff, The University of Chicago (United States)

13 New Developments

Gert de Lange, SRON Netherlands Institute for Space Research (Netherlands) 